\title{
A comparative trial of liver biopsy needles
}

\author{
MC BATESON 1 , D HOPWOOD ${ }^{2}$, HLD DUGUID ${ }^{3}$, AND IAN AD BOUCHIER 1 \\ From the ${ }^{1}$ University Department of Medicine, and ${ }^{2}$ University Department of Pathology, Ninewells \\ Hospital, Dundee, and the ${ }^{3}$ Department of Cytology, Dundee Royal Infirmary, UK
}

SUMmARY A sheathed needle (Tru-Cut) was compared with a suction biopsy needle (Menghini) in a randomised prospective trial over 18 months to determine whether the former offered any special advantages in routine percutaneous liver biopsy. Seventy-seven consecutive biopsies were performed by a single operator. Although biopsy fragmentation was commoner with the suction needle, the length and volume of the largest core obtained was similar to results with the sheathed needle. Cytology provided useful additional information with the Menghini technique. The suction needle was repeatedly reusable and considerably cheaper than the sheathed needle, which may be used once only.

Liver biopsy is a useful diagnostic procedure for which a variety of techniques is in use. A recently introduced innovation is the sheathed needle (Tru-Cut), employed also for breast, kidney, and prostate biopsy. This needle is claimed to yield a better success rate in cirrhosis, ${ }^{1}$ though this has not been fully established. It was considered important to assess any advantages of this new needle over the standard Menghini suction technique.

\section{Procedure}

Patients referred for liver biopsy were randomly allocated to either Menghini or Tru-Cut needle methods. The techniques of biopsy employed the right intercostal approach, either as described by Sherlock $^{2}$ for the Menghini needle, or the revised manufacturer's method for the Tru-Cut needle. The Menghini technique was very rapid, whereas the Tru-Cut technique required several seconds' penetration of the liver.

The needles were matched as far as possible for diameter. The Menghini needle was $1.90 \times 70 \mathrm{~mm}$. The Tru-Cut needle was $2.05 \times 114 \mathrm{~mm}$. A maximum of two attempts was permitted with either needle through a single skin puncture. An estimated $40 \mathrm{~mm}$ liver penetration was made at each attempt. The length of tissue obtained was measured immediately after biopsy, and the cores were then immersed in formol saline before embedding in paraffin and sectioning for histology with the usual staining techniques. The fluid aspirate obtained

Received for publication 2 August 1979 with the Menghini needle was sent for cytology using Papanicolaou and Giemsa stains. The size of the largest core was estimated by calculating the volume from the expression $\pi r^{2} h$, where $r$ was the internal radius of the cutting needle $(0.80 \mathrm{~mm}$ with Menghini needle, $0.86 \mathrm{~mm}$ with Tru-Cut needle) and $h$ was the length of the largest core.

The patients had a screen of blood tests before biopsy and were required to have a haemoglobin greater than $10 \mathrm{~g} / \mathrm{dl}$, platelet count greater than $100 \times 10^{9} / 1$, and a prothrombin time not more than 2 seconds greater than the control. The blood group was determined and serum was saved for possible cross-matching. After biopsy patients were kept lying in bed until the following morning. Pulse and blood pressure were measured four times in the first hour and thereafter hourly until the patient fell asleep at night.

If patients complained of local or shoulder-tip pain an injection of pentazocine $30 \mathrm{mg}$ was given. A chest radiograph was performed on the day after biopsy to exclude pneumothorax.

\section{Results}

The clinical diagnoses necessitating liver biopsy are given in Table 1.

The Menghini needle was used in 36 patients and the Tru-Cut needle in 41. Tissue adequate for diagnosis was obtained in 73 patients. Cirrhosis or liver fibrosis was found in 20 patients, fatty liver in 14 , other abnormalities in 26 , and normal appearances in 13 (Table 2). Fragmentation of the sample occurred in 17 Menghini and six Tru-Cut needle 
Table 1 Clinical diagnosis before liver biopsy

\begin{tabular}{lcc}
\hline Diagnosis & Menghini & Tru-Cut \\
\hline Alcoholic liver disease & 24 & 22 \\
Active chronic liver disease & 4 & 3 \\
Haemochromatosis & 1 & 0 \\
Cholestasis & 2 & 3 \\
Gilbert's syndrome & 2 & 3 \\
Other (includes morbid obesity, & 3 & 10 \\
$\quad$ carcinomatosis, hepatic vein & & \\
$\quad$ thrombosis, porphyria) & 36 & 41 \\
Total & & \\
\hline
\end{tabular}

biopsies $\left(\mathbf{P}<0.01, \chi^{2}\right)$. However, the volume of the largest core obtained was the same with the Menghini and Tru-Cut needles both in cirrhosis and fibrosis $\left(42.2 \pm 5.4 v 35.7 \pm 3.2 \mathrm{~mm}^{3}\right)$ and in the rest $\left(41.9 \pm 3.6 \vee 35.0 \pm 1.8 \mathrm{~mm}^{3}\right)$. If the volumes or lengths of all the fragments obtained were considered, the Menghini needle yielded one-third more tissue.

All but eight of the patients with suspected alcoholic liver disease had abnormal biopsies: in all six for whom cytology was available from a Menghini biopsy there was evidence of liver disease with necrotic liver cells, variability of nuclear size, and excess lymphocytes. One patient also had a suspected hepatoma. Histology showed only necrotic tissue in a $22 \mathrm{~mm}$ core, but cytology demonstrated hepatoma cells, and the diagnosis was subsequently confirmed at necropsy.

A second attempt at liver biopsy was necessary with the Menghini needle in five patients and with the Tru-Cut needle in six. Four of these repeat punctures failed to yield tissue, one with the Menghini and three with the Tru-Cut needle.

Pain requiring analgesia occurred in nine patients with the Menghini needle and in six patients with the Tru-Cut needle. A single dose of pentazocine was sufficient therapy in most. Two patients developed more serious complications, both after Menghini needle biopsies: one with alcoholic hepatitis developed cholangitis, and one with ascending cholangitis developed biliary peritonitis. There was no evidence of significant haemorrhage or of pneumothorax.

Table 2 Results of liver biopsy

\begin{tabular}{lcc}
\hline Histology & Menghini & Tru-Cut \\
\hline Cirrhosis/Fibrosis & 10 & 10 \\
Fatty liver & 4 & 10 \\
Hepatitis, hepatoma, and other & 12 & 14 \\
$\quad$ abnormal & 9 & 4 \\
Normal & 1 & 3 \\
Failure & 36 & 41 \\
Total & & \\
\hline
\end{tabular}

\section{Discussion}

Percutaneous liver biopsy has been performed extensively as a diagnostic aid since its description in $1923 .^{3}$ The equipment used recently has usually involved either a suction needle technique ${ }^{4-6}$ or the sheathed Tru-Cut needle, which has also been widely used. ${ }^{6}$ There has been no rigorous prospective comparison of different needles, though the Tru-Cut needle was introduced for liver biopsy with the claim that it yielded more satisfactory tissue samples, especially in cirrhosis. However, not all patients thought likely to have cirrhosis on clinical grounds prove to have the disease. The poor correlation of blood 'liver function' tests and liver histology was itself a major indication for resorting to a liver biopsy.

The success of the liver biopsy was assessed in this study by the volume of the largest core obtained, calculated from its length measured immediately after the procedure. This method avoided delays in fixation. It also had the advantage over weighing that it did not depend on density, which will be different in pathological conditions such as fatty liver or fibrosis. Fragmentation of the sample was confirmed as a commoner feature of suction biopsy, but even so a satisfactory sample was obtained with the Menghini needle in all but one case.

The diameter of the needle used was determined by the standard size of the Tru-Cut needle. Although there is some suggestion that smaller diameter needles may be safer, ${ }^{7-9}$ this has not been proved. Since more satisfactory samples after a single attempt may be expected with larger needles, and the complication rate is strongly correlated with the number of attempts, ${ }^{6}$ a carefully designed prospective trial would be required to provide a satisfactory answer to the question of safety.

Pain occurred more frequently in our series than in others reported. In one study, 12 out of 140 patients had pain, ${ }^{4}$ and in another 45 out of $1000 .{ }^{6}$ Pain is a highly subjective clinical phenomenon, and the incidence in retrospective series may not provide an accurate estimate of discomfort experienced. Also, in our series two patients developed more severe complications which were both recognised hazards of liver biopsy and did not clearly relate to the type of needle used.

The failure of a second biopsy to yield tissue in four of our patients probably reflected the restriction of the procedure to two punctures. Even with up to 11 attempts a failure rate of $1.9 \%$ has been recorded. ${ }^{6}$

We could show no advantage for the Tru-Cut needle over the Menghini needle when comparable needle diameter and depth of liver penetration were employed. The cost of the disposable Tru-Cut 
needle was (and remains) much more than that of the repeatedly reusable Menghini needle: $£ 4 \cdot 16$ and $£ 1.62$ respectively for the needles used in this study. Even if the Menghini needle was discarded after a single use, and allowing for the cost of syringe and saline, the Tru-Cut needle still costs twice as much. The greater expense cannot be justified on diagnostic grounds.

\section{References}

${ }^{1}$ Rake M O, Murray-Lyon I M, Ansell I D, Williams R. Improved liver-biopsy needle. Lancet 1969; 2: 1283.

${ }^{2}$ Sherlock S. Diseases of the Liver and Biliary System. London: Blackwells, 1975.

${ }^{3}$ Bingel A. Úber die Parenchympunktion der Leber. Verh Dtsch Ges Inn Med 1923; 35: 210-212.

${ }^{4}$ Greenwald R, Chiprut R O, Schiff E R. Percutaneous aspiration liver biopsy using a large-caliber disposable needle. Am J Dig Dis 1977; 22: 1109-1114.

${ }^{5}$ Knauer C M. Percutaneous biopsy of the liver as a procedure for outpatients. Gastroenterology 1978; 74: $101-102$.

${ }^{6}$ Perrault J, McGill D B, Ott B J, Taylor W F. Liver biopsy: complications in 1,000 patients. Gastroenterology 1978; 74: 103-106.

${ }^{7}$ Lundquist A. Liver biopsy with a needle of $0.7 \mathrm{~mm}$ outer diameter. Acta Med Scand 1970; 188: 471-474.

${ }^{8}$ Menghini G. One-second biopsy of the liver-problems of its clinical application. New Engl J Med 1970; 283: 582-585.

${ }^{9}$ Menghini G, Lauro G, Caraceni M. Some innovations in the technic of the one-second needle biopsy of the liver. Am J Gastroenterol 1975; 64: 175-180.

Requests for reprints to: Dr M C Bateson, Department of Medicine, Ninewells Hospital and Medical School, The University of Dundee, Dundee DD1 9SI, UK.

It had been hoped that, as from January 1980, references in all papers would be given in the numbered 'Vancouver' style. However, in the interests of contributors who had submitted manuscripts before the general directive was published, there will be some papers with the Harvard style of references in the first two or three issues of 1980 .

This should not be construed as an encouragement to authors to continue to submit papers in the old style.-Ed. 